\title{
Estresse e Burnout no Trabalho em Oncologia Pediátrica: Revisão Integrativa da Literatura
}

\author{
Stress and Burnout at Work in Pediatric Oncology: \\ an Integrative Literature Review
}

El Estrés y el Agotamiento en el Trabajo en Oncología Pediátrica: una Revisión de la Literatura

Ana Flavia dos Santos \& Manoel Antônio dos Santos

Universidade de São Paulo

http://dx.doi.org/10.1590/1982-370300462014 
Resumo: Profissionais da oncologia pediátrica enfrentam estressores psicossociais no desempenho de suas atividades laborais que os tornam vulneráveis ao burnout. Este estudo teve por objetivo apresentar uma revisão integrativa da literatura relacionada ao estresse e burnout em profissionais da oncologia pediátrica. O levantamento bibliográfico foi realizado nas bases LILACS, MedLine e PsycINFO com análise da produção científica nacional e internacional relativa ao período de 1998 a 2012. Os resultados apontaram para uma produção científica restrita na área. Houve predominância de estudos voltados à identificação de fatores estressores. Enfermeiros constituíram a população mais frequentemente investigada. Os achados mostram a necessidade de ampliação dos estudos que abordem a relação entre estresse ocupacional e aspectos psicossociais, considerando a relevância desses fatores que perpassam a vivência dos profissionais que cuidam da criança com câncer.

Palavras-chave: Profissionais. Stress ocupacional. Psico-oncologia pediátrica. Revisão de literatura.

Abstract: In performing their activities, pediatric oncology professionals encounter psychosocial stressors, making them vulnerable to burnout. This study aimed to present an integrative literature review associated with stress and burnout in pediatric oncology professionals. The review was performed using LILACS, MedLine, and PsycINFO databases for analyzing national and international scientific literature published in 1998-2012. The results revealed a restricted scientific literature. Studies that aimed at identifying stress factors were prevalent; nurses comprised the most investigated class of subjects. The findings highlighted the need for further studies that address the association between occupational stress and psychosocial factors underlying the experience of health professionals who take care of children with cancer.

Keywords: Health personnel. Occupational stress. Pediatric psycho-oncology. Literature review.

Resumen: Profesionales de la oncología pediátrica se enfrentan a factores de estrés en el desempeño de sus actividades de trabajo que los hace vulnerables al agotamiento. Este estudio tuvo como objetivo presentar una revisión integradora de la literatura relacionada con el estrés y el burnout en los profesionales pediátricos oncológicos. La revisión de la literatura se realizó en LILACS, MedLine y PsycINFO con el análisis de la producción científica nacional e internacional en el período de 1998-2012. El corpus se compone de 10 artículos, seleccionados según criterios de inclusión y exclusión previamente establecidos. Los resultados muestran predominio de los estudios dirigidos a identificar los factores de estrés. La población de enfermeras fue la más frecuentemente investigada. Los resultados revelan la necesidad de realizar más estudios para abordar la relación entre el estrés laboral y los aspectos psicosociales, teniendo en cuenta la importancia de estos factores que subyacen a la experiencia de los profesionales que se ocupan de los niños con cáncer.

Palabras clave: Personal de salud. Stres ocupacional. Psico-oncología pediátrica. Revisión de la literatura.

O conceito de estresse foi primeiramente utilizado por Selye (1956), que o caracterizou como uma Síndrome Geral de Adaptação (SGA), decorrente de um evento que exige esforço do indivíduo em termos de adaptação. Esse evento ambiental, denominado estressor, desencadeia a ruptura da homeostase interna do indivíduo, alterando a capacidade do organismo de manter sua constância. O estresse pode se manifestar em três fases: (1) alarme, reação de alerta que prepara o indivíduo para luta ou fuga, mediante a ativação de mecanismos homeostáticos; (2) resistência, tentativa de restabelecer o equilíbrio interno por meio de uma ação reparadora, o que exige dispêndio de energia para consumar a adaptação, tornando o indivíduo suscetível a doenças; e (3) exaustão, etapa caracterizada pelo esgotamento das reservas de energia física e psicológica, momento em que se manifestam as doenças, que podem inclusive ser fatais ou incapacitantes (Lipp, 2004; Lipp \& Malagris, 1998, 2001). 
Lipp (2000) ampliou o modelo proposto por Selye, ao incorporar a fase de quase-exaustão, situada entre as fases de resistência e de exaustão, momento em que há um enfraquecimento do organismo que, ao não conseguir se adaptar ou resistir ao estressor, torna-se vulnerável ao aparecimento de doenças.

Segundo Lazarus e Folkman (1984), a percepção do indivíduo diante de uma determinada situação e a interpretação que ele dá ao evento estressor são de suma importância para o desencadeamento da reação de estresse. Essa percepção e interpretação, elaboradas pelo indivíduo, determinam a resposta de enfrentamento (coping) à situação, conceito desenvolvido pelos referidos autores. A situação pode ser avaliada como excessiva ou estressora, na medida em que excede a capacidade do organismo de assimilá-la a partir da mobilização de esforços cognitivos e comportamentais voltados à redução do estressor (Silva \& Santos, 2010).

As manifestações de estresse podem ocorrer em variados contextos, exigindo uma adaptação do indivíduo ao agente estressor. No âmbito da assistência ao paciente oncológico tem-se observado, no decorrer das últimas duas décadas, uma preocupação com a investigação da relação entre estresse e condições de trabalho, destacando-se o construto do estresse ocupacional (Bond, 1994; Emery, 1993).

Considerando especificamente o estresse no contexto ocupacional, uma das linhas investigativas mais consistentes nesse campo focaliza o burnout, conceito que caracteriza uma síndrome psicológica produzida em resposta aos estressores interpessoais crônicos vivenciados no trabalho (Maslach, Schaufeli, \& Leiter, 2001). A expressão "síndrome de burnout" provém da língua inglesa e deriva do verbo to burn out, que significa queimarse, apagar-se, extinguir-se, o que se mostra perfeitamente adequado para caracterizar a síndrome, uma vez que ela descreve uma série de alterações relacionadas à exposição crônica do trabalhador a eventos estressores associados ao exercício de determinadas profissões. A síndrome de burnout associa-se à exaustão de energia, decorrente de uma má adaptação à exposição prolongada a condições de trabalho estressantes e com elevada carga tensional. Tal síndrome possui três dimensões: (1) esgotamento ou exaustão emocional: sentimentos de estar sobrecarregado e esgotado, que induz um distanciamento cognitivo e emocional em relação ao trabalho; (2) despersonalização: assunção de uma atitude de frieza, indiferença e distanciamento em relação aos colegas e ao contexto de trabalho; e (3) reduzida realização pessoal: sentimento de incompetência, bem como de falta de produtividade e realização no trabalho (Maslach et al., 2001).

O termo burnout, nesse contexto, foi primeiramente utilizado por Freudenberger e, posteriormente, desenvolvido por Maslach (Maslach et al., 2001). A síndrome acomete pessoas que desempenham as mais distintas ocupações. Geralmente se instala em condições de trabalho que requerem elevado grau de contato interpessoal, o que confere maior suscetibilidade a pessoas que atuam com populações específicas, tais como indivíduos gravemente enfermos, crianças, prisioneiros e pessoas incapacitadas ou empobrecidas (Felton, 1998; Santos \& Cardoso, 2010).

Como fontes desencadeadoras do burnout, Lautert (1997) dá relevo às condições de trabalho, bem como às características pessoais dos trabalhadores. As consequências do burnout sobre o desempenho profissional podem se apresentar sob distintas formas: intenção de abandonar o trabalho, absenteísmo e turnover (intensa rotatividade profissional em razão de não conseguir lidar satisfatoriamente com os fatores estressantes a que está exposto em seu cotidiano laboral); queda da eficiência e produtividade para aqueles que continuam no trabalho; reduzida satisfação profissional e baixo nível de comprometimento com a organização; força de trabalho alijada de seu potencial de inovação, dedicação, criatividade e produtividade. Em relação à saúde, as consequências estão associadas às manifestações de estresse e/ou de sintomas indicativos de disfunção no âmbito da saúde mental, como transtorno de ansiedade e depreciação da autoestima, o que pode levar o indivíduo a apresentar uma capacidade reduzida de enfrentar estressores crônicos (Maslach et al., 2001). 
Entre as ocupações que demandam intenso contato interpessoal, destacam-se aquelas relacionadas à área da saúde. Dentre estas, encontra-se a prática profissional no campo da oncologia, reconhecida como área de atuação particularmente estressante devido à alta complexidade dos procedimentos abrangidos, bem como ao envolvimento diário que os profissionais estabelecem com pacientes com doenças potencialmente letais (Whippen \& Canellos, 2001). A exposição contínua e constante ao contato prolongado com situações adversas ou desafiadoras potencializa os fatores estressores inerentes ao manejo de pacientes gravemente adoecidos. Entre os estressores adicionais, presentes na rotina de trabalho dos profissionais que atuam em oncologia, figuram: o aumento das demandas e da complexidade de cuidados exigidos por pacientes e familiares, o lidar com pessoas com doenças graves ou em situação de terminalidade (Kovács, 2008; Schmidt, Gabarra, \& Gonçalves, 2011), que demandam apoio emocional maciço, os limites dos recursos de tratamento, bem como os complexos procedimentos diagnósticos e terapêuticos (Allegra, Hall, \& Yothers, 2005).

Por sua complexidade e especificidade, a oncologia pediátrica é considerada uma especialidade com elevada demanda emocional, na qual os profissionais enfrentam situações de sofrimento e perdas no cotidiano (Spinetta et al., 2000). Pesquisas apontam que enfermeiros da oncopediatria apresentam risco elevado para o estresse ocupacional (Bond, 1994; Emery, 1993). Os estressores vivenciados envolvem, comumente, acompanhar o intenso sofrimento da criança e de seus familiares, tomar conhecimento do agravamento do quadro clínico e de seu desfecho fatal, estar suscetível a cometer erros (Bond, 1994), lidar com as consequências da recidiva ou morte repentina do paciente, incapacidade de oferecer cuidado de qualidade devido à percepção de sobrecarga de trabalho, entre outros fatores adversos (Emery, 1993). Desse modo, o nível de estresse ocupacional parece ser elevado entre os enfermeiros que trabalham em oncologia pediátrica. As limitações do conhecimento médico, somadas às exigências técnicas e ao intenso relacionamento com pacientes e seus familiares, são fontes de desgaste dos profissionais (Valle, 1997). Em relação às demais categorias profissionais, estudo investigou fontes de estresse e de satisfação com o trabalho em médicos, enfermeiros, assistentes sociais e outros especialistas em pediatria que trabalhavam com pais de crianças com câncer (Klassen, Gulati, \& Dix, 2012). Os resultados mostraram que os profissionais de saúde consideram um grande desafio trabalhar com as famílias, por defrontarem muitas vezes com pais exigentes, rudes, com raiva ou que têm visões diferentes sobre o tratamento e os cuidados paliativos. Outros estudos, como Allegra et al. (2005) e Liakopoulou et al. (2008), reportam conclusões semelhantes.

Ambos os construtos - estresse e burnout - ainda que conceitualmente tragam particularidades anteriormente discutidas, vêm sendo investigados relacionados ao contexto ocupacional. O termo estressor, como evento/estímulo que conduz ao estresse, mostra-se relevante nesse campo (Lages et al., 2011). Há que se considerar também que burnout é uma construção recente. Sendo assim, para se identificar a produção científica relativa ao tema, faz-se importante abranger os dois conceitos.

Investigar o estresse e o burnout no âmbito da oncologia pediátrica ganha relevância na medida em que esse contexto de trabalho se caracteriza por elevada carga emocional (Lages et al., 2011; Ramalho, \& Nogueira-Martins, 2007). Em virtude disso, foi publicada tradução das Orientações Psicossociais em Oncologia Pediátrica da Sociedade Internacional de Oncologia Pediátrica (Comitê Psicossocial da Sociedade Internacional de Oncologia Pediátrica, 2000), na qual se apontam, dentre outros aspectos, as causas do burnout que acomete, especificamente, os profissionais de saúde que prestam assistência a crianças e adolescentes com câncer. Dentre os aspectos apontados, é possível assinalar: (1) natureza do trabaIho, tais como lidar cotidianamente com doença grave, de curso imprevisível, e com a situação emocional da criança e de seus familiares; (2) problemas no relacionamento 
entre a equipe multidisciplinar de saúde e o paciente, envolvendo situações tais como: redução do número de trabalhadores, pressões devido à elevada demanda e o tempo insuficiente para se dedicar ao desempenho de tarefas complexas, bem como problemas de comunicação dentro da própria equipe; (3) características pessoais do profissional, tais como dificuldade em solicitar ajuda, envolvimento demasiado ou escasso com o trabalho; e (4) outros aspectos associados, tais como problemas pessoais, desejo de mudar de emprego, baixa remuneração, pressões do sistema de saúde, entre outros. A natureza desse tipo de trabalho, que requer intenso acolhimento emocional, mostra-se tão desgastante para o profissional que o exerce que o documento da SIOP prevê recomendações para prevenir o burnout, considerando aspectos tanto do contexto laboral como extrínsecos a ele.

Considerando o exposto, pode-se afirmar que é relevante voltar a atenção para os problemas decorrentes do desgaste a que o profissional de oncopediatria está sujeito em seu ambiente de trabalho. Porém, como apontaram Liakopoulou et al. (2008) e Klassen et al. (2012), há escassez de estudos na área, de maneira que pouco se sabe sobre o status do conhecimento produzido na interface entre estresse e burnout destes profissionais de saúde. O presente estudo teve por objetivo realizar uma revisão integrativa da literatura relacionada ao estresse e burnout em profissionais da oncologia pediátrica.

\section{Método}

Elegeu-se a revisão integrativa da literatura como estratégia metodológica, considerando que esse tipo de revisão sistemática possibilita sumarizar resultados de pesquisas realizadas anteriormente quanto à temática selecionada, mantendo-se os padrões de clareza, rigor e replicação dos estudos primários (Ganong, 1987). Tal estratégia propõe a discussão crítica dos métodos, fontes, objetivos e resultados, permitindo formular conclusões quanto ao campo de conhecimento delimitado (Broome, 2000).
A operacionalização de uma revisão integrativa pressupõe os seguintes passos: seleção de uma questão temática norteadora e suas hipóteses, estabelecimento de critérios de inclusão e exclusão da amostra, definição de características dos estudos primários, análise de dados, interpretação dos resultados obtidos e apresentação da revisão (Ganong, 1987).

As questões de pesquisa que nortearam este estudo foram: Há na literatura evidências de que profissionais da oncologia pediátrica são vulneráveis ao estresse e burnout? Quais seriam os indicadores relacionados à vulnerabilidade desses profissionais ao estresse e burnout?

O levantamento bibliográfico foi efetuado pela Internet, circunscrevendo a produção científica nacional e internacional ao período de 1998 a 2012, indexada nas bases de dados LILACS, MedLine e PsycINFO. Este período foi delimitado por coincidir com o período imediatamente anterior e posterior à publicação das Orientações Psicossociais em Oncologia Pediátrica (Spinetta et al., 2000). Foram selecionados artigos indexados por meio dos seguintes descritores: "burnout", "estresse", "oncologia", "pediatria" e suas diversas combinações.

Nesta pesquisa bibliográfica foram considerados como critérios de inclusão para busca dos artigos os seguintes parâmetros: (1) artigos publicados entre 1998 e 2012; (2) redigidos na língua portuguesa, inglesa ou espanhola; (3) que disponibilizavam o resumo nas bases indexadoras; (4) que apresentavam resultados empíricos; (5) publicados em periódicos disponibilizados online e na íntegra, seja no site da própria revista ou por meio do sistema de busca SIBI da Universidade de São Paulo, uma rede de serviços que inclui um catálogo eletrônico que franqueia o acesso às bases de dados e conteúdos dos periódicos indexados. Como critérios de exclusão foram estabelecidos os parâmetros: (1) apresentação sob formato de dissertação, tese, capítulo de livro, livro, editorial, resenha, comentário ou crítica; (2) relatos de pesquisa que não guardavam relação direta com a temática investigada. 
A amostra final foi composta por artigos indexados nas bases indexadoras, que preenchiam os critérios de seleção sobre a temática de estresse e burnout em profissionais da oncologia pediátrica. Para operacionalizar a análise elaborou-se um formulário de identificação, que foi preenchido para cada artigo da amostra, permitindo a organização de informações relativas à sua identificação e dos autores, fonte de obtenção e características da publicação, objetivos, características do estudo, análise dos dados e principais resultados, bem como limitações e recomendações sugeridas. Os dados foram organizados segundo os conteúdos investigados e analisados em termos de frequência absoluta (Broome, 2000).

\section{Resultados e Discussão}

Considerando-se a totalidade de artigos capturados a partir das diferentes combinações de unitermos, "stress and oncology" e "burnout and oncology" mostraram-se as mais promissoras, e incluíram os estudos encontrados nas demais combinações. Na base MedLine foram encontradas 1.894 estudos para a combinação "stress and oncology" e 157 para "burnout and oncology", dos quais apenas 21 se afiguraram como específicos do estresse e burnout na oncologia pediátrica. Na base PsycINFO foram localizados 372 e 32 estudos, respectivamente, dos quais apenas dois eram específicos da oncopediatria. $\mathrm{Na}$ base LILACS foram encontrados 25 e 76 estudos, para as respectivas combinações, sendo que apenas 10 atendiam às especificações da área delimitada. Desse modo, chegou-se a um total de 33 publicações recuperadas, dos quais oito se repetiram nas diferentes bases de dados, cinco se referiam a editoriais ou comentários e críticas a outros estudos publicados e dois consistiam em: uma dissertação de mestrado e um trabalho de conclusão de curso. Por conseguinte, descartando-se as repetições e aplicando-se os critérios preestabelecidos, foram excluídas 15 publicações, contabilizando-se, no cômputo final, 18 artigos (Chang, Kicis, \& Sangha, 2007; Dix, Gulati, Robinson, Syed, \& Klassen, 2012; Gallagher \& Gormley, 2009; Hecktman, 2012; Hinds, 2000; Hinds et al., 1998, 2003; Klassen et al., 2012; Liakopoulou et al., 2008; Macpherson,
2008; Morgan, 2009; Mukherjee, Beresford, Glaser, \& Sloper, 2009; Pafaro \& Martino, 2004; Paro, Paro, \& Ferreira, 2005; Ramalho \& Nogueira-Martins, 2007; Spinetta et al., 2000; Zadeh, Gamba, Hudson, \& Wiener, 2012; Zander, Huton, \& King, 2010), que constituíram o corpus da pesquisa, como pode ser visualizado na Tabela.

Conforme pontuam Liakopoulou et al. (2008) e Mukherjee et al. (2009), a literatura na área do burnout na oncologia pediátrica ainda é escassa. Apesar de a preocupação com essa temática remontar à década de 1970 (Spinetta et al., 2000), foi apenas na década de 1990 que houve um interesse mais consistente dos pesquisadores e, ainda assim, em pesquisas mais voltadas para a identificação de estressores vivenciados do que em relação à avaliação do nível de burnout enfrentado (Liakopoulou et al., 2008). Nesse sentido também se pode entender porque foi encontrado, no levantamento bibliográfico realizado, maior número de artigos associados ao estudo do estresse do que à investigação da síndrome de burnout.

Os artigos revisados foram publicados em 11 periódicos diferentes, sendo oito internacionais e três nacionais. Journal of Pediatric Oncology Nursing evidenciouse como a revista com maior número de publicações referente à temática do estresse e do burnout em oncopediatria no período estabelecido para análise (oito estudos). As demais revistas contaram com apenas uma publicação cada: Arquivos de Ciências da Saúde; Clinical Journal of Oncology Nursing; Journal of Oncology Nursing; Journal of Pediatric Hematology/Oncology; Medical and Pediatric Oncology; Pediatric Nursing; Psicologia em Estudo (Maringá); Psychooncology; Revista da Escola de Enfermagem da USP e Support Care Cancer. Pode-se notar que o periódico científico internacional destacado se refere estritamente à área de atuação considerada - a oncologia pediátrica, o que acaba favorecendo a publicação de pesquisas concernentes a esse campo. Tal revista atualiza informações significativas para o conhecimento quanto às práticas oncopediátricas, consistindo em referência 
Tabela. Temas e subtemas encontrados nos artigos revisados, em relação ao problema de pesquisa.

\begin{tabular}{cl}
\hline \multicolumn{1}{c}{ Temas e subtemas } \\
\hline I & Conceituação do estresse \\
II & Conceituação do burnout, causas e prevenção \\
III Impacto do câncer infantil e aspectos envolvidos no \\
cuidado à criança acometida \\
IV $\begin{array}{l}\text { Identificação da área de oncopediatria como } \\
\text { altamente estressante e discriminação dos estressores } \\
\text { mais comuns relatados pelos profissionais da área }\end{array}$
\end{tabular}

V Avaliação do nível de burnout

VI Impacto do estresse e do burnout e as estratégias de enfrentamento utilizadas

VII Propostas de intervenção junto aos profissionais

VIII Modelo de sequência estresse-resposta

IX Aspectos positivos do estresse

X Demandas/exigências e recompensas do trabalho
Artigos

Pafaro e Martino (2004)

Spinetta et al. (2000)

Mukherjee et al. (2009)

Paro et al. (2005)

Hinds et al. (1998)

Hinds (2000)

Spinetta et al. (2000)

Hinds et al. (2003)

Pafaro e Martino (2004)

Chang et al. (2007)

Ramalho e

Nogueira-Martins (2007)

Liakopoulou et al. (2008)

Macpherson (2008)

Morgan (2009)

Mukherjee et al. (2009)

Zander (2010)

Dix et al. (2012)

Hecktman (2012)

Klassen et al. (2012)

Liakopoulou et al. (2008)

Gallagher e Gormley (2009)

Hinds et al. (1998, 2003)

Pafaro e Martino (2004)

Liakopoulou et al. (2008)

Gallagher e Gormley (2009)

Zander et al. (2010)

Hecktman (2012)

Hinds et al. (1998)

Hinds (2000);

Hinds et al. (2003)

Chang et al. (2007)

Macpherson (2008)

Morgan (2009)

Hecktman (2012)

Zadeh et al. (2012)

Hinds et al. (1998, 2003)

Hinds (2000)

Hinds et al. (1998)

Pafaro e Martino (2004)

Dix et al. (2012)

Klassen et al. (2012) específica no cuidado oferecido por profissionais à criança com câncer, área na qual o manejo do estresse situa-se como importante tópico de análise e interesse científico. Outro dado interessante é que cinco das 11 revistas são da área de Enfermagem, o que sugere a 
atenção dedicada à temática por parte dos enfermeiros, uma categoria reconhecidamente vulnerável ao estresse ocupacional.

Quanto ao idioma no qual os artigos foram publicados, constatou-se que 15 foram redigidos na língua inglesa e o restante na língua portuguesa. Vale ressaltar que os periódicos indexados nas bases MedLine e PsycINFO são, em sua maioria, editados originalmente em inglês. Deve-se considerar ainda que estresse e burnout são construtos originalmente desenvolvidos nos Estados Unidos por Selye (1956) e Maslach et al. (2001), respectivamente. Além disso, no que diz respeito à síndrome do burnout, as pesquisas ainda são relativamente recentes no Brasil e, especificamente em relação à oncopediatria, não foi encontrada nenhuma referência anterior aos artigos que compuseram a presente amostra.

Em relação ao número de autores dos artigos selecionados, predominaram aqueles redigidos por um ou três autores (quatro artigos em cada categoria). Em seguida, apareceram artigos elaborados por dois autores (três publicações); dois estudos redigidos por quatro autores, dois artigos escritos-por 10 autores e, por fim elaborados por cinco, 11 ou 12 autores (uma publicação em cada categoria). Destaca-se tanto o impacto negativo que a síndrome de burnout pode ter em relação ao bem-estar da equipe de saúde, quanto o papel fortalecedor que o grupo/equipe pode ter nesse contexto, como estratégia de enfrentamento face aos estressores biológicos ou psicossociais e ao risco de burnout (Maslach et al., 2001). Esses temas configuram problemática de interesse de e para grupos de trabalho, o que aponta para uma perspectiva de interação, alimentada pelo trabalho compartilhado de investigação. Isso, por si só, pode refletir a busca de apoio mútuo, que funciona como estratégia de enfrentamento ao burnout e estresse ocupacional. Ademais, o fato de uma instituição dar suporte a um trabalho de pesquisa sobre o tema já é indicativo de que há valorização dessa questão, independentemente da quantidade de autores envolvidos em cada estudo.
Ao considerar a titulação do primeiro autor das pesquisas que compõem a amostra dos artigos revisados, nove têm doutorado, três artigos foram assinados por mestres, três por alunos de graduação, um por mestrando e um por graduando. Não foi informada a titulação do autor de um dos artigos. Esses dados indicam que, entre os artigos revisados, houve proeminência de investigadores com alto nível de qualificação ou especialização, o que condiz com o esperado em uma área de alta complexidade. Verifica-se que as produções brasileiras foram assinadas por graduandos, mestrandos e mestres, o que parece estar em conformidade com o interesse recente pela abordagem da temática no Brasil, consistindo em uma linha de pesquisa original, mas ainda em fase inicial, daí ser conduzida, muitas vezes, no início da formação do pesquisador em saúde. Assim, a pós-graduação se afigura como importante instância propulsora de qualificação dos pesquisadores e disseminadora de conhecimento na área.

Quanto ao país de origem do primeiro autor de cada artigo, nove dos estudos revisados têm autores oriundos dos Estados Unidos. Três artigos são procedentes do Brasil, três do Canadá e um da Grécia, um da Austrália e um da Grã-Bretanha. Uma vez que o país de origem sinaliza a área geográfica onde se realizam investigações na temática considerada, a predominância de publicações produzidas na América do Norte, sobretudo nos Estados Unidos, pode estar relacionada tanto ao fato de serem uma megapotência científica, como à origem dos construtos estresse e burnout no referido país. Isso provavelmente reflete o fato de que esse é o centro mundial de interesses e investimentos vultosos na área da oncologia pediátrica, o que se reflete na proeminência de pesquisas sobre estresse e burnout dos profissionais que atuam nesse contexto. Ainda que essas linhas de pesquisa sejam recentes em nosso país, nota-se uma participação expressiva da produção nacional em relação a dos outros países nos últimos anos.

Como local de atuação do primeiro autor dos artigos revisados, identificou-se concentração de profissionais lotados em hospital geral 
(nove artigos) e na universidade (nove). Uma vez que se considera que o local de atuação do pesquisador funciona como fonte potencializadora e fomentadora do surgimento de questões de investigação, pode-se supor que a temática do estresse e do burnout no trabalho em oncologia pediátrica seja uma questão de relevância por estar presente no cuidado cotidiano das crianças com câncer. Pesquisas sistematizadas podem funcionar como molas propulsoras para o desenvolvimento de propostas interventivas voltadas ao aprimoramento do contexto de trabalho em questão. A prática hospitalar pode ser mobilizadora de questões de pesquisa, propiciando investigações a respeito dos problemas locais vivenciados no cotidiano pelo profissional de saúde. Quanto ao trabalho dos autores na universidade, pode-se inferir que, sendo esse contexto um locus de produção de conhecimento por excelência, é esperado que haja uma preocupação com questões atuais e de interesse científico, que sirvam de estímulo para o desenvolvimento de pesquisas de interesse clínico.

Quanto ao desenho de pesquisa, foram encontrados 13 estudos subdivididos em não experimentais ou descritivos (10) e experimentais (três). Cinco dos estudos eram de natureza teórica ou documental (revisão). O delineamento metodológico conferido a cada estudo tem relação com a natureza do problema investigado. Estudos descritivos são aqueles que visam a estimar parâmetros, descrevendo suas características e estabelecendo relações entre variáveis. Os estudos teóricos relacionam-se à sistematização e análise da produção científica por meio da pesquisa bibliográfica. No presente estudo de revisão observou-se predominância dos estudos descritivos, com enfoque quantitativo, o que indica uma preocupação em observar e descrever os aspectos do fenômeno estudado, bem como enfatizar os atributos mensuráveis da experiência (Polit, Beck, \& Hungler, 2004).

No que concerne aos instrumentos empregados para alcançar os objetivos propostos nas pesquisas, verificou-se uma diversidade de ferramentas, variando de um a sete instrumentos por estudo revisado, com predomínio do uso de questionários (sete estudos) em comparação com a técnica de entrevista semiestruturada (cinco). Os demais estudos não fizeram uso de instrumento, sendo cinco teóricos. Considerando essa diversidade, foram computados 17 instrumentos diferentes. Stressor Scale for Pediatric Oncology Nurses foi o instrumento mais utilizado (três estudos), em consonância com o objetivo de identificar os estressores presentes na prática do enfermeiro em oncologia pediátrica. $\mathrm{O}$ Maslach Burnout Inventory, um instrumento internacionalmente conhecido e utilizado para avaliação da síndrome de burnout, foi utilizado em apenas duas investigações. Mukherjee et al. (2009) criticaram as taxas de respostas obtidas nos estudos publicados e apontaram que as deficiências nas propriedades psicométricas dos instrumentos utilizados para avaliar as fontes de estresse limitam as conclusões extraídas em relação aos aspectos estressantes que perpassam o trabalho em oncologia pediátrica.

Nos estudos revisados foi empregada uma diversidade de estratégias metodológicas. Uma vez que a escolha do delineamento metodológico deve ser congruente com o objetivo proposto no estudo, pôde-se identificar uma variedade de objetivos ou mesmo ausência de definição consolidada - que, no entanto, pode estar em vias de estruturação - quanto aos instrumentos mais adequados para a avaliação nessa área do conhecimento.

Considerando os instrumentos privilegiados nos estudos, pode-se deduzir um menor interesse pela identificação do estresse e do burnout em termos de sua mensuração, em comparação com a identificação de fatores estressores no trabalho. Cabe destacar a ênfase dada nos estudos à avaliação do enfrentamento (coping), do apoio social e da satisfação profissional.

Em relação aos profissionais que compõem a população investigada nos diferentes estudos selecionados, a temática foi abordada predominantemente em enfermeiros, considerados em 17 artigos, sendo 11 dedicados exclusivamente a esses profissionais, ao passo que os demais abrangeram profissionais de 
saúde de diversas categorias. Cabe notar que, em um dos estudos, as profissões não foram discriminadas.

A enfermagem constitui a profissão dominante no que diz respeito ao interesse das pesquisas na área do estresse e do burnout, sendo considerada, muitas vezes, como aquela categoria que fornece a essência do cuidado (Murofuse, Abranches, \& Napoleão, 2005). Os enfermeiros constituem grande parte do contingente de profissionais da instituição hospitalar, consistindo na linha de frente no atendimento à criança com câncer (Pafaro \& Martino, 2004). Além disso, por dedicarem mais tempo ao contato direto com os pacientes, os enfermeiros se encontram cronicamente expostos a situações estressantes, devido às diversificadas responsabilidades que lhe são atribuídas e às particularidades do trabalho desenvolvido em hospitais. Esse trabalho se caracteriza por acentuada falta de organização e parca participação nas decisões, além da excessiva burocratização e das precárias condições do sistema de saúde (Lautert, 1997).

Por outro lado, outras categorias profissionais acabam sendo negligenciadas pelas pesquisas, o que limita as conclusões que podem ser extraídas ao se examinar o estresse laboral em oncologia pediátrica. Isso resulta em um desconhecimento em relação às diferentes realidades ocupacionais que compõem a equipe de saúde. O estudo de revisão de Mukherjee et al. (2009) também apontou limitações no poder de generalização dos dados em razão de os estudos se concentrarem quase que exclusivamente em enfermeiros. Diversificados, os profissionais das categorias que compõem a equipe multidisciplinar executam diferentes funções dependendo de cada formação especializada; desempenham papéis variados no gerenciamento e assumem responsabilidades específicas no cuidado das crianças e de suas famílias. Assim, pode-se supor que as categorias profissionais estão suscetíveis a diferentes tipos de eventos e situações estressantes, de acordo com sua inserção no trabalho. Um dos poucos estudos que compararam as experiências de médicos e enfermeiros comprovou que existem diferenças entre os estressores vivenciados pelos profissionais dessas categorias (Papadatou, Bellali, Papazoglou, \& Petraki, 2002).

Vale destacar que as pesquisas descritivas, que consideram outras profissões que atuam na área da oncologia pediátrica como suscetíveis ao estresse e ao burnout, são mais recentes, datando de 2007 em diante. Isso pode indicar uma tendência atual de ampliação da abordagem desses aspectos psicossociais, que estão presentes em atividades profissionais de outra natureza que não exclusivamente a da Enfermagem. A identificação dos temas e subtemas encontrados nas publicações revisadas permite definir os eixos que nortearam a produção dos estudos e a elaboração dos artigos (Tabela).

A maior parte dos estudos revisados (dez) apoiou-se na evidência de que a área de oncopediatria é altamente estressante, sendo, portanto, necessário identificar os estressores mais frequentemente associados ao estresse a que estão submetidos os profissionais que atuam nessa especialidade. Também foram abordados aspectos tais como tipos de intervenção (oito estudos) e impacto do estresse e do burnout (cinco), bem como as estratégias de enfrentamento utilizadas pelos profissionais (seis) e demandas/exigências e recompensas do trabalho (dois) também foram abordados.

Também foi possível reconhecer o modelo teórico-conceitual no qual os diferentes estudos relatados nos artigos desta revisão se basearam, o que permite identificar alguns caminhos a serem seguidos por novos estudos na área. Assim, é necessário que investigações futuras explorem a relação entre construtos tão complexos como vulnerabilidade ao estresse e burnout, o que possibilitará identificar os fatores e processos envolvidos na produção desses fenômenos. A maior parte dos estudos pautou-se na compreensão da área da oncopediatria por meio do modelo conceitual do estresse (Tabela). Cinco estudos pautaram-se no modelo do burnout (Gallagher \& Gormley, 2009; Liakopoulou et al., 2008; Mukherjee et al., 2009; Ramalho \& Nogueira-Martins, 2007; Spinetta et al., 2000). Em seguida, 
o modelo de sequência estresse-resposta constituiu-se como um dos mais utilizados, concentrando uma produção representada na presente revisão por três publicações (Hinds, 2000; Hinds et al., 1998, 2003). Pafaro e Martino (2004) fundamentaram sua contribuição na concepção clássica de estresse proposta por Selye (1956). Um estudo não explicitou a abordagem teórico-conceitual utilizada pelos autores (Paro, Paro, \& Ferreira, 2005).

Chang et al. (2007), Macpherson (2008) e Zadeh et al. (2012) realizaram estudos de intervenção. O primeiro explorou o efeito do papel da enfermeira de apoio clínico (EAC) sobre o estresse dos enfermeiros de uma unidade de oncopediatria de um hospital canadense. A EAC dá apoio à equipe de primeira linha que lida com os desafios da prática, buscando reduzir as tensões relacionadas à atividade clínica. Macpherson (2008) examinou o impacto de sessões de contação de histórias - sobre pacientes que morreram - em pares de enfermeiros de oncologia pediátrica. Contar histórias é uma atividade utilizada para a produção de significados por profissionais de saúde que cuidam de crianças em processo de morte, de modo que possam lidar com suas experiências de luto e tristeza. Por sua vez, Zadeh et al. (2012) desenvolveram um programa de promoção do bem-estar para a equipe de enfermeiros, combinando aproximações ao trabalho e ensino. Os estudos de Morgan (2009) e Hecktman (2012) também apresentaram propostas de intervenção.

Nos estudos que compuseram o corpus desta revisão integrativa predominou a concepção de estresse em comparação com a de burnout, reproduzindo a tendência observada em outros levantamentos bibliográficos. É preciso ter em mente que o termo burnout é um construto mais recente do que o de estresse, estando menos disseminado e popularizado. Faz-se importante ressaltar, no entanto, que não são conceitos que se sobrepõem. A vulnerabilidade ao estresse se associa a questões amplas da vida, não estando associada a uma causa específica, pois se refere tanto a questões objetivas como subjetivas. Já o burnout se relaciona intimamente a questões do trabalho. No entanto, essa concepção vem sendo atualmente criticada e questionada, uma vez que a sintomatologia que define a síndrome do esgotamento encontra-se inteiramente presente em outras condições, como os transtornos psicopatológicos - por exemplo, depressão. Assim, Milan (2007) ressalta que a diferença entre estresse e burnout reside no apontamento das condições de trabalho como desencadeadoras do processo, o que por si só não justifica a criação de um novo conceito. A crítica recai na nova terminologia, e não no estudo da problemática observada, uma vez que se reconhece que se trata de um fator fundamental para o incremento da qualidade de vida de profissionais dedicados às atividades de cuidado. Nesse sentido, nota-se concentração de estudos voltados à identificação dos estressores no trabalho, ou seja, de possíveis fatores desencadeantes do burnout associados à prática profissional.

As definições do estresse e do burnout foram pouco contempladas nos artigos, o que pode dificultar a compreensão quanto à fundamentação teórica que sustenta os estudos. Pode-se destacar ainda que, dos artigos selecionados, três indicaram os aspectos positivos associados ao estresse, já presentes quando da formulação clássica do conceito por Selye (1956), que pontuou que o estresse pode ter tanto efeito positivo quanto negativo sobre o organismo. Ainda que os efeitos prejudiciais sejam mais enfatizados (distress), o estresse pode agir beneficamente (eustress), sendo inclusive necessário para preparar o indivíduo para a ação. Assim, percebe-se ainda nos estudos o uso do termo "estresse" com uma conotação negativa, associado a situações graves de perda ou adoecimento.

Com base nesses pressupostos, foram identificados os objetivos dos estudos revisados. Os objetivos gerais, tais como apresentados pelos artigos que compõem a amostra, foram agrupados e organizados segundo cinco eixos, a saber: (1) testagem do modelo de sequência estresse-resposta (três estudos); (2) avaliação da manifestação de estresse / burnout (três); (3) avaliação do impacto de uma intervenção em relação ao estresse experimentado (quatro); (4) identificação da vivência ocupacional 
dos profissionais da área da oncopediatria (sete); e (5) diretrizes para o reconhecimento e prevenção ao burnout (um).

Alguns estudos se dedicaram à identificação da vivência ocupacional dos profissionais da área, fazendo referência ao modelo conceitual do estresse. Nessa vertente, pode-se destacar o modelo de sequência estresse-resposta, que se baseia na identificação de quatro componentes inter-relacionados: os estressores para os profissionais da oncopediatria, suas respostas (biológicas ou psicossociais) aos estressores, as consequências (negativas ou positivas) e os mediadores que configuram o contexto no qual essa sequência é disparada (Hinds et al., 1998). Esse modelo certamente é o mais frequentemente utilizado na investigação dessas questões, propiciando a identificação completa de diferentes aspectos envolvidos no desencadeamento do estresse. Vale ressaltar que quatro das dez publicações mais atuais, considerando o período de 2008 a 2012, são estudos de revisões, indicando uma possível preocupação recente com o mapeamento do estado da arte.

Questões relacionadas à avaliação do estresse/ burnout e das estratégias de intervenção em função do estresse e da vivência ocupacional apontam para diferentes perspectivas de investigação, nas quais a identificação não só de fatores estressores, como também o levantamento de aspectos gratificantes do trabalho são aspectos importantes, bem como a avaliação de possíveis mudanças nos padrões de estresse favorecidas por processos interventivos aplicados. Além disso, destaca-se o interesse pela sistematização do quadro de burnout, com objetivo de oferecer instrumentalização que permita a identificação do quadro e forneça sustentação a possíveis ações preventivas a serem implementadas. Uma vez que o modelo de sequência estresse-resposta também visa a identificar os estressores laborais em enfermeiros da oncopediatria, assim como os estudos voltados à vivência ocupacional, a identificação dos fatores potencializadores do estresse laboral figura como o objetivo mais reiterado nos artigos.
Além disso, a avaliação do estresse também se encontra associada à testagem do modelo de sequência estresse-resposta, que foi o segundo objetivo mais estabelecido pelos estudos (cinco). Cabe destacar que o modelo de sequência estresse-resposta se volta à identificação de diferentes componentes, tais como enfrentamento e satisfação com o trabalho, o que mostra que a avaliação do estresse se encontra intimamente relacionada a esses aspectos. Desse modo, pode-se compreender que existe um interesse em investigar os aspectos psicossociais mais amplos, o que implica utilizar uma abordagem integral na avaliação do estresse.

Ao identificar, nos artigos revisados, os pressupostos teóricos, os temas e os objetivos dos estudos, foi possível identificar a produção científica acerca do estresse e do burnout que afetam o trabalho em oncologia pediátrica, reconhecendo sua evolução, bem como as tendências dessa área de conhecimento. Para ampliar a compreensão desse contexto, organizou-se uma síntese a partir da aglutinação dos dados apresentados nos artigos selecionados. Foram elaboradas quatro categorias temáticas, que serão apresentadas a seguir.

\section{Vivências de estresse ocupacional em profissionais da oncologia pediátrica}

Spinetta et al. (2000) apresentam o contingente dos profissionais de saúde e, mais especificamente, daqueles que oferecem cuidado a crianças, como especialmente vulnerável ao burnout, com impacto negativo para o indivíduo, para a equipe, bem como para os beneficiários do cuidado oferecido - a criança e sua família. Paro et al. (2005) caracterizaram a atuação em Enfermagem como propiciadora de sofrimento e estresse. Ramalho e Nogueira -Martins (2007) identificaram suscetibilidade semelhante em diferentes profissionais da área oncopediátrica.

Zander et al. (2010) afirmam que o trabalho na área impõe uma sobrecarga tanto pessoal como profissional, tendo em vista estressores 
adicionais específicos ao campo. Zadeh et al. (2012) apontam que enfermeiros pediátricos, devido à especificidade de seu trabalho, têm risco aumentado para estresse e exaustão emocional. Especificamente no contexto do transplante de medula óssea, a maioria dos enfermeiros apresentou nível de esgotamento emocional de moderado a alto e nível de despersonalização moderado (Gallagher \& Gormley, 2009). O estudo de Dix et al. (2012) corroborou a intensa sobrecarga que incide sobre esses profissionais, apesar de os avanços tecnológicos na área terem oportunizado crescentes taxas de sobrevida dos pacientes submetidos ao tratamento. $\mathrm{Na}$ contramão desses achados, Mukherjee et al. (2009) afirmam que a evidência de estresse e burnout entre profissionais da oncologia pediátrica é extremamente limitada, embora haja indícios qualitativos de que enfermeiros dessa área vivenciam estressores, tanto específicos como adicionais, não experimentados pelos profissionais que atuam em serviços destinados a pacientes adultos.

Hinds et al. (1998) e Hinds (2000) verificaram vivência de estresse relacionado ao trabalho de enfermeiros, sendo mais grave entre aqueles mais jovens, que demandam maior esforço para responder ao estressor. Hinds et al. (2003) encontraram enfermeiros da área da oncopediatria com alto a moderado nível de estresse. Pafaro e Martino (2004), ao investigarem enfermeiros com dupla jornada de trabalho em hospital de oncologia pediátrica, constataram que a maioria dos profissionais apresentava manifestações de estresse, com predomínio de sintomas na fase de resistência e de sintomatologia psicológica. Liakopoulou et al. (2008), em estudo com médicos e enfermeiros oncopediátricos, verificaram níveis similares de burnout para os dois grupos profissionais.

\section{Fatores estressantes no trabalho de profissionais da oncopediatria}

Mukherjee et al. (2009) e Klassen et al. (2012) destacam que a identificação de estressores relacionados ao trabalho no contexto da oncopediatria é limitada. No entanto, Mukherjee et al. (2009) apontam a necessidade de compreender melhor os estressores relacionados ao trabalho nesse contexto, uma vez que esse conhecimento pode contribuir para evitar os impactos negativos. Para desenvolver intervenções apropriadas e efetivas de apoio à equipe, é necessário acumular muito mais conhecimento acerca dos níveis de burnout e morbidade psiquiátrica experimentados por todos os membros da equipe multidiscipliar da Oncopediatria, bem como os fatores que contribuem para os desfechos negativos, como o esgotamento emocional e a síndrome do burnout.

Segundo Spinetta et al. (2000), no contexto da oncopediatria destacam-se como causas do burnout: (1) a natureza do trabalho realizado, que exige uma rotina baseada no tratamento de doença grave, constante ameaça à vida ou presença da morte, cuidado a crianças com saúde comprometida, exigência de aparentar "estar bem" diante dos pacientes e dos familiares; (2) a interação com o ambiente de trabalho, as expressões de autoritarismo, tensão e hostilidade entre colegas de trabalho; falta de apoio dos colegas quando são enfrentadas as maiores dificuldades; pressões da elevada demanda e pouco tempo de elaboração das experiências vividas; e (3) características pessoais, como falta de preparo profissional, expectativa elevada quanto ao trabalho de um membro da equipe, não compartilhamento de ideias, não usufruir do apoio social oferecido pelos colegas.

Ramalho e Nogueira-Martins (2007) identificaram os seguintes fatores estressores: falta de recursos de ajuda disponibilizados ao profissional, fraca estrutura administrativa, falhas na coordenação do grupo, baixa remuneração, sobrecarga de trabalho, falta de comunicação e de compartilhamento entre colegas da equipe, falta de reconhecimento do trabalho, características da doença e do tratamento, e a morte das crianças. Entre enfermeiros oncopediátricos verificaram-se os estressores: contato constante com a morte, sentimento de impotência e descrença nas medidas terapêuticas, falta de estrutura de suporte, falta de recursos de apoio psicológico, vivência de conflitos e 
ansiedades nas relações equipe-familiar, desgaste emocional, exigência de intenso nível de relacionamento interpessoal e atendimento às necessidades emocionais da criança e do familiar (Paro et al., 2005), acompanhamento do sofrimento do paciente e da família, convívio cotidiano com a morte, trabalhar com colegas cujo envolvimento com o paciente se mostra inadequado (Hinds, 2000) e possibilidade de cometer erros (Chang et al., 2007).

Klassen et al. (2012) detectaram aspectos desafiadores ao lidar com as famílias na perspectiva dos profissionais, tais como: dificuldade em lidar com comportamentos inapropriados dos pais, visões divergentes quanto ao tratamento e aos cuidados paliativos, e a comunicação de más notícias.

Dix et al. (2012) verificaram que os profissionais relacionam muitos aspectos do trabalho como sendo tanto recompensadores como de sobrecarga, corroborando o estudo de Morgan (2009), realizado especificamente no contexto dos cuidados paliativos para crianças com câncer. Para este autor, os profissionais têm dificuldades em atuar nos cuidados paliativos, bem como em lidar com sentimentos suscitados quando se veem fazendo algo por obrigação em oposição aos seus princípios e convicções pessoais, o que pode levar a reações de ansiedade e agravamento do estresse laboral.

Mukherjee et al (2009) identificaram os seguintes estressores na atuação dos enfermeiros: sobrecarga de trabalho, trabalhar com criança em sofrimento e/ou morrendo, contato com os pais, acompanhar frequentemente a morte de pacientes, entre outros. Zander et al. (2010) apontam estressores específicos, tais como o confronto com dilemas moras e éticos, perdas constantes, doenças e regimes de tratamento altamente complexos e o próprio relacionamento com pacientes e familiares. Segundo Hecktman (2012), se o profissional não é capaz de identificar o estresse crônico a que está submetido ou se ele deparar com enormes dificuldades de implementação de estratégias para reduzir as fontes de sofrimento no trabalho, pode ser progressivamente levado à exaustão.

\section{Fatores gratificantes no trabalho}

Como fatores gratificantes no trabalho, Ramalho e Nogueira-Martins (2007) identificaram o relacionamento interpessoal e o vínculo afetivo prolongado, estabelecidos com a criança e sua família, o gostar do que se faz e observar o resultado do tratamento da criança. Também foram encontrados os fatores: alta satisfação com o trabalho, forte coesão grupal e elevado nível de comprometimento organizacional, indicando que o estresse não apenas resulta em consequências negativas (Hinds et al., 1998, 2003). Outros fatores de satisfação encontrados foram: ajudar pacientes e familiares a obterem os melhores resultados e oferecer conforto por meio de suas ações ou de seus colegas de trabalho (Hinds et al., 1998). Esses elementos podem funcionar como fatores de proteção em relação ao estresse laboral, contribuindo, assim, para aumentar a resistência à síndrome do burnout. De acordo com Klassen et al. (2012), a preservação do contato próximo com a família do paciente e os vínculos de longa duração podem funcionar como fatores de proteção durante toda a jornada familiar.

Segundo Dix et al. (2012), os profissionais da oncologia pediátrica descreveram o exercício de seu ofício como significativo, gratificante e capaz de levá-los à realização. Gallagher e Gormley (2009) sugerem que sistemas de apoio podem afetar a satisfação e o sentimento de realização de enfermeiros que trabalham em unidades de transplante de medula óssea.

\section{Prevenção e enfrentamento do estresse e do burnout}

As questões de prevenção se situam em dois âmbitos: (1) mudanças de aspectos negativos do ambiente do trabalho, para favorecer o encontro, troca e discussão entre os profissionais da equipe, tais como: recrutamento e treinamento cuidadosos, desenvolvimento de um clima de trabalho favorável e de respaldo eficiente, adoção de estratégias de controle de estressores, disseminação da flexibilidade 
e autonomia no trabalho, incentivo ao apoio social entre colegas, entre outros; e (2) modificações de aspectos do comportamento do indivíduo que predispõem ao burnout, a fim de resgatar o senso de autoeficácia, tais como: aprendizado quanto aos limites de energia e tempo a serem investidos nas atividades laborais, evitação de excessivo envolvimento emocional, manutenção do equilíbrio entre vida pessoal e profissional, respeito ao tempo de lazer, reconhecimento precoce dos sintomas de estresse, psicoterapia, entre outros recursos de enfrentamento (Spinetta et al., 2000).

Chang et al. (2007) constataram que a presença da enfermeira de apoio clínico esteve associada à redução da intensidade do estresse em enfermeiros, principalmente nos profissionais mais jovens. Macpherson (2008) identificou impacto positivo, no sentido da redução da aflição e tristeza sentidas pelo profissional no trabalho, de uma intervenção que consistia em receber e prover apoio em pares em sessões de contação de histórias de pacientes que foram a óbito. Zander et al. (2010) investigaram o papel da resiliência como fator mediador do estresse entre profissionais da oncologia pediátrica. São reconhecidos como importantes fatores de proteção ao estresse e ao burnout: ter clareza em relação à função laboral exercida (Liakopoulou et al., 2008), desfrutar de satisfação profissional (Hinds et al., 1998; Liakopoulou et al., 2008) e contar com suporte profissional e de colegas de trabalho (Liakopoulou et al., 2008; Paro et al., 2005; Ramalho \& Nogueira-Martins, 2007).

Para Morgan (2009), reconhecer as necessidades dos profissionais auxilia nas intervenções voltadas a assegurar melhores condições de cuidado e maior satisfação profissional. Hecktman (2012) propôs um rol de recomendações, considerando as intervenções existentes, de modo a criar um conjunto de dados para pesquisas subsequentes. Zander et al. (2010) propuseram um levantamento das intervenções voltadas à redução de desfechos negativos, como o burnout. Zadeh et al. (2012) discutiram a importância de se implementar um programa voltado ao bem-estar dos enfermeiros, de modo a auxiliá-los no manejo dos eventos estressores de seu trabalho.

De modo geral, os resultados dos estudos revisados responderam aos objetivos propostos, propiciando identificar a produção científica que se ocupa prioritariamente em desvendar os fatores estressantes a que estão expostos os profissionais da oncopediatria, principalmente os enfermeiros. No entanto, os dados não se restringiram a esses fatores, pois também foi dedicada atenção a aspectos do coping, satisfação e intervenção, que complementaram a abordagem da temática relacionada ao estresse e ao burnout, ainda que apareçam com menor frequência e destaque nos estudos. Esses aspectos são mais consistentemente apresentados como pressupostos norteadores do que propriamente sob a forma de resultados sistemáticos de pesquisas.

Investigou-se, por fim, se os artigos revisados identificavam as limitações do estudo desenvolvido e/ou ofereciam recomendações futuras quanto a novos direcionamentos de pesquisa. Verificou-se que os estudos apontaram limitações, relacionadas ao tamanho da amostra (Chang et al., 2007; Macpherson, 2008), impossibilidade de avaliação do estresse antes e depois da intervenção (Chang et al., 2007) e baixas correlações entre os resultados devido à fraca correlação encontrada entre os componentes do modelo avaliado (Hinds, 2000; Hinds et al., 1998, 2003). Klassen et al. (2012) indicaram como limitações de seu estudo o recrutamento realizado em apenas quatro centros oncológicos do Canadá e o predomínio de mulheres entre os profissionais entrevistados. Contudo, os autores ressalvam que esse desequilíbrio entre os sexos reflete a natureza da força de trabalho na oncologia pediátrica.

Mukherjee et al. (2009) apontaram, em sua revisão, o número restrito de estudos publicados e concluíram que a capacidade de generalização dos resultados de muitos deles é questionável, devido ao tamanho reduzido das amostras utilizadas, taxas de respostas modestas que provavelmente 
subestimam o nível real de estresse e burnout experimentado, deficiência das propriedades psicométricas dos instrumentos empregados para mensurar a morbidade psiquiátrica e o burnout, ou o não uso desses instrumentos padronizados, o recrutamento de sujeitos de associações profissionais, o número restrito de centros investigados (por vezes, único) e o fato de a maior parte das pesquisas ter sido realizada nos Estados Unidos.

Em relação às recomendações apresentadas, 13 estudos indicaram implicações práticas dos resultados, apontando também para a necessidade de desenvolver novas pesquisas na área da oncopediatria ou de intervenção na prática clínica. Dentre esses, quatro indicaram a melhoria das ações na oncopediatria devotadas especificamente ao cuidado do cuidador, sob a forma de suporte técnico, psicológico ou de encontros regulares entre membros da equipe para prover e receber apoio mútuo (Liakopoulou et al., 2008; Macpherson, 2008; Paro et al., 2005; Ramalho \& Nogueira-Martins, 2007). Três estudos sugeriram a necessidade de revisão do modelo utilizado e propuseram um novo paradigma para sua compreensão (Hinds, 2000; Hinds et al., 1998, 2003). Um artigo apontou a necessidade de expandir a amostra para abarcar outros profissionais, pacientes e familiares (Chang et al., 2007).

Ao investigar os cuidados paliativos, Morgan (2009) privilegiou as estratégias utilizadas para reduzir o estresse relacionado ao trabalho. Zander et al. (2010) destacam a necessidade de se aprofundar o estudo sobre resiliência. Mukherjee et al. (2009) recomendam estudos que relacionam recompensa, satisfação, enfrentamento e proteção em relação ao estresse. Hecktman (2012) propõe comparar intervenções, identificando a de melhor custo-benefício e a mais eficaz. Esses achados sugerem que há, da parte dos profissionais que atuam na área da oncopediatria que são autores e coautores dos estudos revisados, um compromisso com o desenvolvimento científico do tema, apesar de os estudos terem uma apreensão pouco crítica em relação aos aspectos metodológicos adotados. Ainda assim, nota-se a preocupação com novos direcionamentos de pesquisa e com a sistematização de modelos de intervenção que resultem em aperfeiçoamentos na realidade, ainda que a forma de promover mudanças seja pouco ou nada descrita nos estudos analisados, estando apenas indicada em alguns.

Mukherjee et al. (2009) afirmam que, devido às limitações identificadas nos estudos disponíveis, a pesquisa atual não fornece evidências sobre o nível de desgaste ou morbidade psiquiátrica na equipe de oncologia pediátrica. Pesquisas robustas e em condições de proprocionar resultados que possam ser generalizáveis são necessárias para identificar a extensão em que se faz necessário intervir para aliviar o estresse $\mathrm{e}$ apoiar as equipes de saúde.

\section{Considerações Finais}

Uma revisão integrativa foi realizada para sintetizar e analisar a produção científica nacional e internacional dedicada ao estresse e burnout em profissionais da oncologia pediátrica, considerando as contribuições dos últimos anos. A caracterização dos estudos revisados permitiu apontar uma produção científica escassa relacionada ao estresse e burnout em profissionais da oncologia pediátrica, sugerindo que se trata de um interesse de pesquisa ainda não consolidado, embora em franca expansão. Desse modo, pode-se inferir o reconhecimento que essa temática tem despertado na era contemporânea. Os enfermeiros constituíram a população mais frequentemente estudada nos trabalhos, havendo lacunas em relação a outras categoriais profissionais, o que impossibilita uma comparação e ampliação do espectro de investigação da realidade ocupacional nessa área.

Os estudos fundamentados na concepção de estresse prevaleceram sobre as investigações do burnout, o que pode ser decorrente de uso mais disseminado do conceito de estresse. $\mathrm{O}$ objetivo mais frequentemente definido pelos estudos foi de identificação de fatores estressantes do trabalho assistencial. Nos estudos analisados constatou-se uma concepção mais 
negativa do que positiva associada ao estresse. Ainda que as questões do enfrentamento e da satisfação com o trabalho tenham sido abordadas, apareceram com menor destaque, sugerindo que se trata de um campo ainda a ser explorado. Propostas de intervenção foram pouco delineadas nos estudos, o que é indicativo da necessidade de ampliar os investimentos em pesquisa nesse aspecto.

Prevaleceram estudos desenvolvidos no contexto internacional. Os achados sugerem que a produção brasileira encontra-se em estágio incipiente, sendo em geral conduzida por pesquisadores com menor titulação acadêmica quando comparados aos autores estrangeiros. Pode-se inferir que esse campo de investigação no país ainda está estabelecendo as bases para seu desenvolvimento e, portanto, encontra-se distante do processo de consolidação.

O fato de vários estudos terem sido conduzidos por autores vinculados ao contexto hospitalar indica que os profissionais da oncologia pediátrica estão interessados em compreender melhor as dificuldades vivenciadas no cuidado intensivo à criança com câncer. Essas dificuldades, se não forem identificadas e superadas - ou, pelo menos, acolhidas e mitigadas - podem potencializar a vulnerabilidade do profissional ao estresse e ao burnout. Por outro lado, o próprio contexto de trabalho faz emanarem questões de pesquisa a serem esclarecidas ou solucionadas por meio de investigação específica, cujos resultados podem favorecer a elaboração de propostas de ações preventivas e interventivas a serem colocadas em prática no próprio local.

Os objetivos dos estudos, de modo geral, foram alcançados e apontaram-se limitações e implicações para a atenção em saúde. Destaca-se a indicação do modelo de sequência de estresse-resposta que, apesar de dominar os estudos na área, apresenta limitações, o que indica a necessidade de incorporação de novos modelos para a compreensão do estresse que afeta os profissionais da oncopediatria.

Algumas limitações deste estudo podem ser mencionadas, como o número reduzido de artigos recuperados e o fato de o recorte temporal da revisão ser circunscrito a alguns anos, não permitindo examinar a evolução do estado da arte ao longo de um período de tempo mais extenso. Considerando essas limitações, esta revisão não permite descrever ou extrair conclusões acerca do panorama atual do conhecimento na área. A contribuição pretendida pelo estudo é no sentido de explorar um campo ainda pouco conhecido na Oncopediatria e que, ao ser objeto de novas investigações, poderá ter implicações importantes na prática profissional, além de oferecer potenciais subsídios teóricos.

A partir da análise empreendida, aponta-se a necessidade de ampliação dos estudos a fim de abarcar outras categoriais profissionais, que não apenas a dos enfermeiros, e que se comprometam com aspectos psicossociais que se encontram associados à concepção de estresse, tais como enfrentamento, qualidade de vida, satisfação com o trabalho e comprometimento organizacional. É preciso abarcar de modo mais consistente a relação entre estresse e aspectos psicossociais que perpassam a experiência do profissional de saúde que cuida da criança com câncer, a fim de que sejam delineadas propostas de intervenção efetivas para o manejo de situações estressoras encontradas no cotidiano dos profissionais da oncopediatria. 


\section{Ana Flavia dos Santos}

Mestre pela Universidade de São Paulo, São Paulo - SP. Brasil.

E-mail: anaflavia_angel@hotmail.com

\section{Manoel Antônio dos Santos}

Doutorado em Psicologia Clínica pela Universidade de São Paulo, São Paulo - SP. Brasil. Docente da Universidade de São Paulo, São Paulo - SP.

\section{Endereço para envio de correspondência:}

Universidade de São Paulo, Faculdade de Filosofia Ciências e Letras de Ribeirão Preto, Departamento de Psicologia e Educação. Avenida Bandeirantes, 3900, Monte Alegre. CEP: 14040901. Ribeirão Preto - SP. Brasil.

Os autores agradecem o apoio financeiro recebido do Conselho Nacional de Desenvolvimento Científico e Tecnológico (CNPq) para a realização deste estudo, mediante concessão de Bolsa de Produtividade em Pesquisa, nível 1B, ao segundo autor.

Recebido 04/08/2014, Reformulação 06/02/2015, Aprovado 11/03/2015. 
Referências
Allegra, C. J., Hall, R., \& Yothers, G. (2005). Prevalence of burnout in the U.S. oncology community: results of a 2003 survey. Journal of Oncology Practice, 1(4), 140-147.

Bond, D. C. (1994). The measured intensity of work-related stressors in pediatric oncology nursing. Journal of Pediatric Oncology Nursing, 11(2), 44-54.

Broome, M. E. (2000). Integrative literature reviews for the development of concepts. In B. L. Rodgers, \& K. A. Knafl (Orgs.), Concept development in nursing: foundations, techniques, and applications (2nd ed., pp. 231-250). Philadelphia, PA: W. B. Saunders.

Chang, A., Kicis, J., \& Sangha, G. (2007). Effect of the clinical support nurse role on workrelated stress for nurses on an inpatient pediatric oncology unit. Journal of Pediatric Oncology Nursing, 24(6), 340-349.

Comitê Psicossocial da Sociedade Internacional de Oncologia Pediátrica (SIOP). (2000). Orientações psicossociais em oncologia pediátrica (L. P. C. Françoso, \& E. R. M. Valle, trads.). Ribeirão Preto: Grupo de Apoio à Criança com Câncer (GACC).

Dix, D., Gulati, S., Robinson, P., Syed, I., \& Klassen, A. (2012). Demands and rewards associated with working in pediatric oncology: a qualitative study of Canadian health care providers. Journal of Pediatric Hematology/Oncology, 34(6), 430-435.

Emery, J. E. (1993). Perceived sources of stress among pediatric oncology nurses. Journal of Pediatric Oncology Nursing, 10(3), 87-92.

Felton, J. S. (1998). Burnout as a clinical entity: its importance in health care workers. Occupational Medicine, 48(4), 237-250.

Gallagher, R., \& Gormley, D. K. (2009). Perceptions of stress, burnout, and support systems in pediatric bone marrow transplantation nursing. Clinical Journal Oncology Nursing, 13(6), 681-685.

Ganong, L. H. (1987). Integrative reviews of nursing research. Research in Nursing and Health, 10(1), 1-11.

Hecktman, H. M. (2012). Stress in pediatric oncology nurses. Journal of Oncology Nursing, 27(2), 356-361.
Hinds, P. S. (2000). Testing the stress-response sequence model in pediatric oncology nursing. Journal of Pediatric Oncology Nursing, 17(2), 59-68.

Hinds, P. S., Sanders, C. B., Srivastava, D. K., Hickey, S., Jayawardene, D., Milligan, M. et al. (1998). Testing the stress-response sequence model in pediatric oncology nursing. Journal of Pediatric Oncology Nursing, 28(5), 1146-1157.

Hinds, P. S., Srivastava, D. K., Randall, E. A., Green, A., Stanford, D., Pinlac, R., et al. (2003). Testing the revised stress-response sequence model in pediatric oncology nurses. Journal of Pediatric Oncology Nursing, 20(5), 213-232.

Klassen, A., Gulati, S., \& Dix, D. (2012). Health care providers' perspectives about working with parents of children with cancer: a qualitative study. Journal of Pediactric Oncology Nursing, 29(2), 92-97.

Kovács, M. J. (2008). Desenvolvimento da tanatologia: estudos sobre a morte e o morrer. Paidéia, 18(41), 457-468.

Lages, M. G. G., Costa, M. A. O., Lopes, T. R., Amorim, F. C. S., Araujo Neto, A. P., Nascimento, I. R. D., et al. (2011). Estratégias de enfrentamento de enfermeiros frente ao paciente oncológico pediátrico. Revista Brasileira de Cancerologia, 57(4), 503-510.

Lautert, L. (1997). O desgaste profissional: uma revisão da literatura e implicações para a enfermeira. Revista Gaúcha de Enfermagem, 18(2), 83-93.

Lazarus, R. S., \& Folkman, S. (1984). Stress, appraisal and coping. New York, NY: Sringer.

Liakopoulou, M., Panaretaki, I., Papadakis, V., Katsika, A., Sarafidou, J., Laskari, H., et al. (2008). Burnout, staff support, and coping in pediatric oncology. Support Care Center, 16(1), 143-150.

Lipp, M. E. N. (2000). Manual do Inventário de sintomas de stress para adultos de Lipp. São Paulo, SP: Casa do Psicólogo.

Lipp, M. E. N. (Org.) (2004). O stress no Brasil: Pesquisas avançadas. Campinas, SP: Papirus. 
Lipp, M. E. N., \& Malagris, L. N. (1998). Manejo de estresse. In B. Rangé (Org.), Psicoterapia comportamental e cognitiva: teoria, pesquisa e aplicações (pp. 279-292). São Paulo: Psy.

Lipp, M. E. N., \& Malagris, L. N. (2001). O stress emocional e seu tratamento. In B. Rangé (Org.), Psicoterapia comportamental e cognitiva: teoria, pesquisa e aplicações (pp. 475-489). Porto Alegre: ArtMed.

Macpherson, C. F. (2008). Peer-supported storytelling for grieving pediatric oncology nurses. Journal of Pediatric Oncology Nursing, 25(3), 148-163.

Maslach, C., Schaufeli, W. B., \& Leiter, M. P. (2001). Job burnout. Annual Review Psychology, 52, 397-422.

Milan, L. R. (2007). A síndrome de burnout: realidade ou ficção? Revista da Associação Médica Brasileira, 53(1), 1-12.

Morgan, D. (2009). Caring the dying children: Assessing the needs of the pediatric palliative care nurse. Pediatric Nursing, 35(2), 86-90.

Mukherjee, S., Beresdford, B., Glaser, A., \& Sloper, P. (2009). Burnout, psychiatric morbidity, and work-related sources of stress in paediatric oncology staff: a review of the literature. Psychooncology, 18(10), 1019-1928.

Murofuse, N. T., Abranches, S. S., \& Napoleão, A. A. (2005). Reflexões sobre estresse e burnout e a relação com a Enfermagem. Revista Latino-americana de Enfermagem, 13(2), 255-261.

Pafaro, R. C., \& Martino, M. M. F. (2004). Estudo do estresse do enfermeiro com dupla jornada de trabalho em um hospital de oncologia pediátrica de Campinas. Revista da Escola de Enfermagem da USP, 38(2), 152-160.

Papadatou, D., Bellali, T., Papazoglou, I., \& Petraki, D. (2002). Greek nurse and physician grief as a result of caring for children dying of cancer. Pediatric Nursing, 28(4), 345-353.

Paro, D., Paro, J., \& Ferreira, D. L. M. (2005). $\mathrm{O}$ enfermeiro e o cuidar em oncologia pediátrica. Arquivos de Ciências da Saúde, 12(3), 143-149.
Polit, D. F., Beck, C. T., \& Hungler, B. P. (2004). Análise quantitativa. In D. F. Polit, C. T. Beck, \& B. P. Hungler (Orgs.), Fundamentos de pesquisa em enfermagem: Métodos, avaliação e utilização (pp. 167-198). 5a ed. Porto Alegre: ArtMed.

Ramalho, M. A. N., \& Nogueira-Martins, M. C. F. (2007). Vivências de profissionais de saúde da área de oncologia pediátrica. Psicologia em Estudo, 12(1), 123-132.

Santos, A. F., \& Cardoso, C. L. (2010). Profissionais de saúde mental: manifestação de stress e burnout. Estudos de Psicologia (Campinas), 27(1), 67-74.

Schmidt, B., Gabarra, L. M., \& Gonçalves, J. R. (2011). Intervenção psicológica em terminalidade e morte: relato de experiência. Paidéia, 21(50), 423-430.

Selye, H. (1956). The stress of life. New York: Longmans.

Silva, G., \& Santos, M. A. (2010). Stressors in breast cancer post-treatment: a qualitative approach. Revista Latino-Americana de Enfermagem, 18(4), 688-695.

Spinetta, J. J., Jankovic, M., Arush, M. W. B., Eden, T., Epelman, C., Greenberg, M. L., et al. (2000). Guidelines for the recognition, prevention, and remediation of burnout in health care professionals participating in the care of children with cancer: report of the SIOP working committee on psychosocial issues in pediatric oncology. Medical and Pediatric Oncology, 35(2), 122-125.

Valle, E. R. M. (1997). Câncer infantil: Compreender e agir. Campinas, SP: Psy.

Whippen, D. A., \& Canellos, G. P. (2001). Burnout syndrome in the practice of oncology: results of a random survey of 1,000 oncologists. Journal of Clinical Oncology, 9(10), 1916-1920.

Zadeh, S., Gamba, N., Hudson, C., \& Wiener, L. (2012). Taking care of care providers: A wellness program for pediatric nurses. Journal of Pediatric Oncology Nursing, 29(5), 294-299.

Zander, M., Hutton, A., \& King, L. (2010). Coping and resilience factors in pediatric oncology nurses. Journal of Pediatric Oncology Nursing, 27(2), 94-108. 